\title{
Crystal structure of tetrakis(acetonitrile)zinc(II) sulfate, $\mathrm{Zn}\left(\mathrm{C}_{2} \mathrm{H}_{3} \mathrm{~N}\right)_{4} \mathrm{SO}_{4}$
}

\author{
H.-L. Yangl, F. Yang ${ }^{\mathrm{II}}$ and H.-L. Zhu*,II \\ I Xiangfan University, Department of Chemistry, Xiangfan, Hubei, 441000 P. R. China \\ II Wuhan University of Science and Engineering. Department of Chemistry, Wuhan, 430073 P. R. China \\ III Fuyang Normal College, Department of Chemistry, Fuyang, Anhui, 236041 P. R. China
}

Received February, 22, 2004, accepted in revised form and available on-line August 14, 2004; CCDC no. $1267 / 1261$

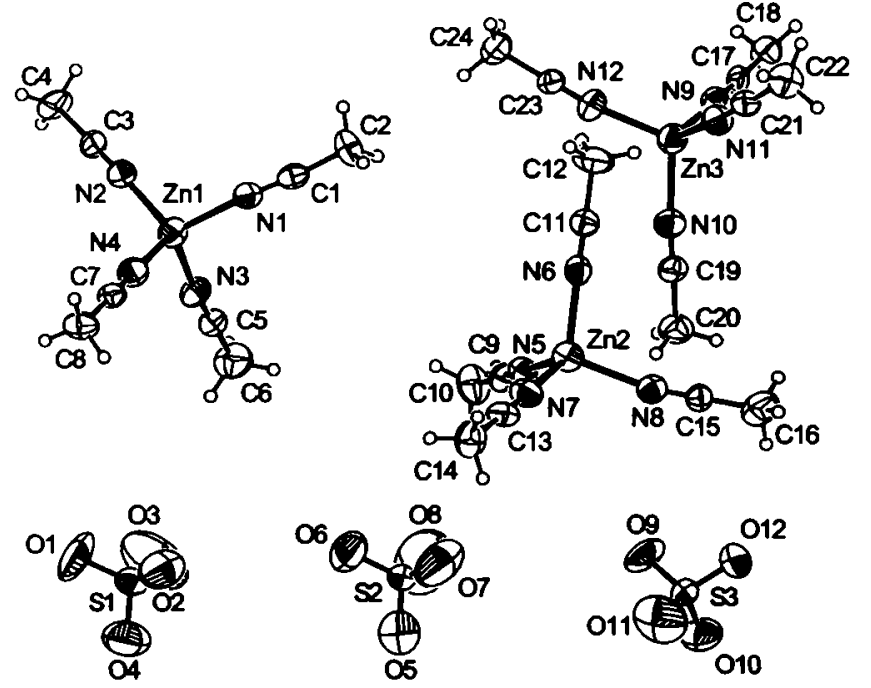

Abstract

$\mathrm{C}_{8} \mathrm{H}_{12} \mathrm{~N}_{4} \mathrm{O}_{4} \mathrm{SZn}$, orthorhombic, $P$ na2 ${ }_{1}$ (no. 33), $a=24.25(1) \AA$, $b=8.456(3) \AA, c=20.743(8) \AA, V=4253.9 \AA^{3}, Z=12$, $R_{\mathrm{gt}}(F)=0.050, w R_{\text {ref }}\left(F^{2}\right)=0.131, T=298 \mathrm{~K}$.

\section{Source of material}

All reagents and solvents were used as purchased without further purification. C, $\mathrm{H}$ and $\mathrm{N}$ elemental analyses were performed on a Perkin-Elmer elemental analyzer.

An aqua-acetonitrile solution of $\mathrm{ZnSO}_{4} \cdot 6 \mathrm{H}_{2} \mathrm{O}$ was poured into a Teflon vessel till $30 \%$ of its volume was filled. Then the vessel was placed in a stainless steel tank to perform hydrothermal treatment. The crystallization reaction was performed at $160^{\circ} \mathrm{C}$ under autogenous pressure for $24 \mathrm{~h}$. After the autoclave was cooled and depressurized, colorless slab-shaped crystals were deposited at the bottom of the Teflon vessel. After filtering and washing with aqueous acetonitrile solution for three times, they were dried under $\mathrm{CaCl}_{2}$ (yield $66 \%$ ). Elemental analysis: found - C, $29.38 \%$; $\mathrm{H}, 3.81 \% ; \mathrm{N}, 17.12 \%$; calc. for $\mathrm{C}_{8} \mathrm{H}_{12} \mathrm{~N}_{4} \mathrm{O}_{4} \mathrm{SZn}-\mathrm{C}, 29.50 \%$; $\mathrm{H}, 3.71 \% ; \mathrm{N}, 17.20 \%$.

\section{Discussion}

The asymmetric unit of the title structure being isotypic to [1-4] consists of three mononuclear tetraacetonitrile cations and three sulfate anions. In each cation, the central zinc(II) atom is fourcoordinated by nitrogen atoms from four acetonitrile ligands. The average $\mathrm{Zn}-\mathrm{N}$ bond lengths are 1.999(8) $\AA$ for $\mathrm{Znl}, 2.033$ (7) $\AA$ for $\mathrm{Zn} 2$, and 1.996 (8) $\AA$ for $\mathrm{Zn} 3$, respectively. The angles around each metal vary from $106.2(3)^{\circ}$ to $113.0(3)^{\circ}$ for $\mathrm{Znl}$, from $105.4(2)^{\circ}$ to $111.5(3)^{\circ}$ for $\mathrm{Zn} 2$, or from $107.3(3)^{\circ}$ to $111.9(3)^{\circ}$ for

\footnotetext{
* Correspondence author (e-mail: hailiang_zhu@fync.edu.cn)
}

$\mathrm{Zn} 3$, indicating that the polyhedra around the metal centers are slightly distorted tetrahedra. The Flack $x$ parameter of $0.10(2)$ confirms the absolute structure.

Table 1. Data collection and handling.
Crystal:

Wavelength:

$\mu$ :

Diffractometer, scan mode:

$2 \theta_{\max }$ :

$N(h k l)_{\text {measured, }} N(h k l)_{\text {tunique: }}$

Criterion for $I_{\mathrm{obs}}, N(\mathrm{hkl})_{\mathrm{gt}}$ :

$N$ (param) hefined:

Programs: colorless slab, size $0.30 \times 0.35 \times 0.45 \mathrm{~mm}$ Mo $K_{\alpha}$ radiation $(0.71073 \AA)$ $18.90 \mathrm{~cm}^{-1}$

Siemens SMART CCD, $\varphi / \omega$ $50.06^{\circ}$

20950, 7197

$I_{\text {obs }}>2 \sigma\left(I_{\text {obs }}\right), 3964$

499

SADABS [1], SHELXTL [2]
Table 2. Atomic coordinates and displacement parameters (in $\AA^{2}$ ).

\begin{tabular}{|c|c|c|c|c|c|}
\hline Atom & Site & $\boldsymbol{x}$ & $y$ & $z$ & $U_{\text {iso }}$ \\
\hline $\mathbf{H}(2 \mathrm{~A})$ & $4 a$ & 1.1463 & 1.0489 & 0.3707 & 0.122 \\
\hline H(2B) & $4 a$ & 1.1327 & 1.1023 & 0.3001 & 0.122 \\
\hline$H(2 C)$ & $4 a$ & 1.1610 & 0.9384 & 0.3125 & 0.122 \\
\hline$H(4 A)$ & $4 a$ & 0.8866 & 1.0906 & 0.5409 & 0.103 \\
\hline H(4B) & $4 a$ & 0.8632 & 0.9218 & 0.5560 & 0.103 \\
\hline$H(4 C)$ & $4 a$ & 0.8317 & 1.0338 & 0.5084 & 0.103 \\
\hline$H(6 A)$ & $4 a$ & 0.8553 & 1.1037 & 0.1871 & 0.136 \\
\hline$H(6 B)$ & $4 a$ & 0.8485 & 0.9376 & 0.1544 & 0.136 \\
\hline$H(6 C)$ & $4 a$ & 0.9017 & 1.0383 & 0.1418 & 0.136 \\
\hline $\mathrm{H}(\mathbf{8 A})$ & $4 a$ & 0.9713 & 0.2219 & 0.2994 & 0.099 \\
\hline $\mathrm{H}(8 \mathrm{~B})$ & $4 a$ & 0.9209 & 0.2237 & 0.3467 & 0.099 \\
\hline $\mathrm{H}(8 \mathrm{C})$ & $4 a$ & 0.9811 & 0.2159 & 0.3741 & 0.099 \\
\hline$H(10 A)$ & $4 a$ & 0.0416 & -0.2953 & 0.4679 & 0.115 \\
\hline $\mathrm{H}(10 \mathrm{~B})$ & $4 a$ & 0.0116 & -0.2845 & 0.5347 & 0.115 \\
\hline $\mathrm{H}(10 \mathrm{C})$ & $4 a$ & 0.0762 & -0.2888 & 0.5317 & 0.115 \\
\hline$H(12 A)$ & $4 a$ & 0.1754 & 0.5418 & 0.6598 & 0.134 \\
\hline$H(12 B)$ & $4 a$ & 0.1501 & 0.4163 & 0.7069 & 0.134 \\
\hline $\mathrm{H}(12 \mathrm{C})$ & $4 a$ & 0.1203 & 0.5788 & 0.6964 & 0.134 \\
\hline$H(14 A)$ & $4 a$ & -0.1282 & 0.6100 & 0.5083 & 0.122 \\
\hline$H(14 B)$ & $4 a$ & -0.1489 & 0.4672 & 0.5500 & 0.122 \\
\hline$H(14 C)$ & $4 a$ & -0.1530 & 0.4599 & 0.4747 & 0.122 \\
\hline$H(16 A)$ & $4 a$ & 0.1470 & 0.6193 & 0.3550 & 0.129 \\
\hline$H(16 B)$ & $4 a$ & 0.1169 & 0.5244 & 0.3005 & 0.129 \\
\hline $\mathrm{H}(16 \mathrm{C})$ & $4 a$ & 0.1707 & 0.4565 & 0.3318 & 0.129 \\
\hline $\mathrm{H}(18 \mathrm{~A})$ & $4 a$ & 0.4900 & 0.2891 & 0.7032 & 0.110 \\
\hline $\mathrm{H}(18 \mathrm{~B})$ & $4 a$ & 0.4901 & 0.1959 & 0.6378 & 0.110 \\
\hline $\mathrm{H}(18 \mathrm{C})$ & $4 a$ & 0.4832 & 0.1047 & 0.7031 & 0.110 \\
\hline$H(20 A)$ & $4 a$ & 0.2221 & 0.2268 & 0.4513 & 0.108 \\
\hline$H(20 B)$ & $4 a$ & 0.1667 & 0.2332 & 0.4884 & 0.108 \\
\hline $\mathrm{H}(20 \mathrm{C})$ & $4 a$ & 0.1967 & 0.0717 & 0.4802 & 0.108 \\
\hline $\mathrm{H}(22 \mathrm{~A})$ & $4 a$ & 0.2777 & 0.8846 & 0.6308 & 0.096 \\
\hline $\mathrm{H}(22 \mathrm{~B})$ & $4 a$ & 0.3256 & 0.8846 & 0.6816 & 0.096 \\
\hline $\mathrm{H}(22 \mathrm{C})$ & $4 a$ & 0.2640 & 0.8796 & 0.7047 & 0.096 \\
\hline $\mathrm{H}(24 \mathrm{~A})$ & $4 a$ & 0.1898 & 0.3122 & 0.8726 & 0.126 \\
\hline$H(24 B)$ & $4 a$ & 0.2251 & 0.1620 & 0.8886 & 0.126 \\
\hline $\mathrm{H}(24 \mathrm{C})$ & $4 a$ & 0.1689 & 0.1442 & 0.8520 & 0.126 \\
\hline
\end{tabular}


Table 3. Atomic coordinates and displacement parameters (in $\AA^{2}$ ).

\begin{tabular}{|c|c|c|c|c|c|c|c|c|c|c|}
\hline Atom & Site & $x$ & $y$ & $z$ & $U_{11}$ & $U_{22}$ & $U_{33}$ & $U_{12}$ & $U_{13}$ & $U_{23}$ \\
\hline $\mathrm{Zn}(1)$ & $4 a$ & $0.96036(4)$ & $0.7985(2)$ & $0.34889(4)$ & $0.0605(7)$ & $0.0633(8)$ & $0.0540(8)$ & $0.0037(6)$ & $0.0008(5)$ & $0.0032(9)$ \\
\hline $\operatorname{Zn}(2)$ & $4 a$ & $0.04329(4)$ & $0.2871(1)$ & $0.50597(5)$ & $0.0609(7)$ & $0.0596(7)$ & $0.0632(8)$ & $0.0011(6)$ & $-0.0013(6)$ & $-0.0004(9)$ \\
\hline $\operatorname{Zn}(3)$ & $4 a$ & $0.29149(4)$ & $0.3044(1)$ & $0.67127(6)$ & $0.0589(5)$ & $0.0673(6)$ & $0.0591(6)$ & $0.0000(5)$ & $0.0009(5)$ & $0.000(1)$ \\
\hline $\mathbf{N}(1)$ & $4 a$ & $1.0375(3)$ & $0.8824(8)$ & $0.3418(3)$ & $0.061(4)$ & $0.057(4)$ & $0.048(4)$ & $-0.003(4)$ & $-0.002(3)$ & $-0.004(4)$ \\
\hline$N(2)$ & $4 a$ & $0.9232(3)$ & $0.8855(8)$ & $0.4274(3)$ & $0.057(4)$ & $0.060(5)$ & $0.049(4)$ & $-0.003(4)$ & $-0.003(3)$ & $0.002(4)$ \\
\hline $\mathbf{N}(3)$ & $4 a$ & $0.9191(3)$ & $0.8822(8)$ & $0.2713(3)$ & $0.063(4)$ & $0.058(5)$ & $0.049(4)$ & $0.011(4)$ & $0.001(3)$ & $0.006(4)$ \\
\hline$N(5)$ & $4 a$ & $0.044 I(3)$ & $0.0517(8)$ & $0.5071(4)$ & $0.070(5)$ & $0.052(5)$ & $0.064(5)$ & $-0.005(4)$ & $-0.013(4)$ & $0.001(6)$ \\
\hline$N(6)$ & $4 a$ & $0.0868(2)$ & $0.3740(8)$ & $0.5817(3)$ & $0.051(4)$ & $0.054(5)$ & $0.054(4)$ & $-0.003(3)$ & $0.002(3)$ & $0.009(4)$ \\
\hline$N(7)$ & $4 a$ & $-0.0357(2)$ & $0.3706(8)$ & $0.5086(3)$ & $0.049(4)$ & $0.063(4)$ & $0.055(4)$ & $-0.008(3)$ & $-0.001(4)$ & $-0.008(4)$ \\
\hline$N(8)$ & $4 a$ & $0.0816(3)$ & $0.3754(8)$ & $0.4247(3)$ & $0.052(4)$ & $0.060(5)$ & $0.054(4)$ & $0.005(4)$ & $-0.004(3)$ & $0.000(4)$ \\
\hline $\mathbf{N}(9)$ & $4 a$ & $0.3696(2)$ & $0.2350(7)$ & $0.6722(4)$ & $0.041(3)$ & $0.073(4)$ & $0.064(4)$ & $0.005(3)$ & $-0.004(5)$ & $-0.003(4)$ \\
\hline$N(10)$ & $4 a$ & $0.2524(3)$ & $0.2318(9)$ & $0.5928(4)$ & $0.059(5)$ & $0.059(6)$ & $0.060(5)$ & $0.004(3)$ & $-0.004(4)$ & $-0.006(4)$ \\
\hline $\mathbf{N}(11)$ & $4 a$ & $0.2913(2)$ & $0.5440(7)$ & $0.6699(4)$ & $0.056(3)$ & $0.051(4)$ & $0.051(3)$ & $-0.002(4)$ & $-0.005(4)$ & $0.009(6)$ \\
\hline$N(12)$ & $4 a$ & $0.2518(3)$ & $0.2372(8)$ & $0.7508(4)$ & $0.064(5)$ & $0.068(6)$ & $0.051(4)$ & $0.005(4)$ & $0.013(4)$ & $0.003(4)$ \\
\hline$O(2)$ & $4 a$ & $1.0127(2)$ & $0.7089(9)$ & $0.6752(5)$ & $0.088(5)$ & $0.184(8)$ & $0.097(5)$ & $0.022(5)$ & $-0.007(6)$ & $-0.019(7)$ \\
\hline$O(3)$ & $4 a$ & $0.9547(4)$ & $0.908(1)$ & $0.6736(8)$ & $0.23(1)$ & $0.101(7)$ & $0.36(2)$ & $-0.003(7)$ & $-0.07(2)$ & $-0.03(1)$ \\
\hline$O(4)$ & $4 a$ & $0.9320(4)$ & $0.698(1)$ & $0.6229(5)$ & $0.107(7)$ & $0.175(9)$ & $0.124(7)$ & $-0.011(6)$ & $-0.019(6)$ & $-0.047(7)$ \\
\hline$O(5)$ & $4 a$ & $0.6824(5)$ & $0.701(2)$ & $0.7871(5)$ & $0.17(1)$ & $0.23(1)$ & $0.131(9)$ & $-0.080(9)$ & $0.019(7)$ & $-0.012(9)$ \\
\hline$O(6)$ & $4 a$ & $0.6817(4)$ & $0.718(1)$ & $0.8943(4)$ & $0.118(8)$ & $0.178(9)$ & $0.076(5)$ & $-0.020(6)$ & $0.004(5)$ & $0.023(6)$ \\
\hline$O(7)$ & $4 a$ & $0.7565(4)$ & $0.751(2)$ & $0.8340(4)$ & $0.082(7)$ & $0.39(2)$ & $0.096(7)$ & $0.006(8)$ & $0.002(5)$ & $-0.001(8)$ \\
\hline$O(8)$ & $4 a$ & $0.6966(7)$ & $0.908(2)$ & $0.8316(8)$ & $0.39(2)$ & $0.15(1)$ & $0.26(2)$ & $-0.00(1)$ & $0.08(2)$ & $-0.00(1)$ \\
\hline$O(9)$ & $4 a$ & $0.1755(3)$ & $0.783(1)$ & $0.5641(3)$ & $0.074(5)$ & $0.207(9)$ & $0.056(4)$ & $0.010(5)$ & $0.024(4)$ & $-0.038(5)$ \\
\hline$O(10)$ & $4 a$ & $0.1794(3)$ & $0.781(1)$ & $0.4541(4)$ & $0.102(6)$ & $0.139(7)$ & $0.071(5)$ & $-0.004(5)$ & $-0.025(4)$ & $0.003(5)$ \\
\hline$O(11)$ & $4 a$ & $0.2079(4)$ & $0.573(1)$ & $0.5062(6)$ & $0.22(1)$ & $0.068(6)$ & $0.20(1)$ & $0.003(6)$ & $-0.027(9)$ & $0.007(7)$ \\
\hline$O(12)$ & $4 a$ & $0.2593(3)$ & $0.7995(9)$ & $0.5112(4)$ & $0.080(5)$ & $0.135(6)$ & $0.089(5)$ & $-0.045(4)$ & $0.017(4)$ & $-0.019(5)$ \\
\hline$S(1)$ & $4 a$ & $0.95839(8)$ & $0.7623(5)$ & $0.6742(2)$ & $0.058(1)$ & $0.056(2)$ & $0.064(1)$ & $0.0024(9)$ & $-0.006(2)$ & $-0.013(1)$ \\
\hline$S(2)$ & $4 a$ & $0.7037(1)$ & $0.7692(4)$ & $0.8376(2)$ & $0.055(2)$ & $0.053(2)$ & $0.050(2)$ & $-0.005(1)$ & $-0.002(1)$ & $-0.004(1)$ \\
\hline$S(3)$ & $4 a$ & $0.2058(1)$ & $0.7330(4)$ & $0.5098(2)$ & $0.055(2)$ & $0.056(2)$ & $0.045(2)$ & $-0.004(1)$ & $0.004(1)$ & $-0.004(1)$ \\
\hline$C(1)$ & $4 a$ & $1.0806(3)$ & $0.9387(9)$ & $0.3337(3)$ & $0.060(5)$ & $0.047(5)$ & $0.042(5)$ & $0.011(4)$ & $-0.007(4)$ & $-0.003(4)$ \\
\hline$C(2)$ & $4 a$ & $1.1348(4)$ & $1.014(1)$ & $0.3288(6)$ & $0.058(6)$ & $0.083(8)$ & $0.103(9)$ & $-0.025(6)$ & $0.004(6)$ & $0.005(6)$ \\
\hline$C(5)$ & $4 a$ & $0.9005(3)$ & $0.9373(9)$ & $0.2274(4)$ & $0.049(5)$ & $0.045(5)$ & $0.049(5)$ & $0.008(4)$ & $0.004(4)$ & $-0.004(4)$ \\
\hline$C(6)$ & $4 a$ & $0.8744(3)$ & $1.010(1)$ & $0.1733(7)$ & $0.124(8)$ & $0.073(6)$ & $0.075(6)$ & $0.000(6)$ & $-0.014(8)$ & $0.019(8)$ \\
\hline$C(7)$ & $4 a$ & $0.9603(3)$ & $0.433(1)$ & $0.3425(4)$ & $0.055(5)$ & $0.053(6)$ & $0.043(5)$ & $-0.001(5)$ & $0.000(4)$ & $-0.010(6)$ \\
\hline$C(8)$ & $4 a$ & $0.9582(4)$ & $0.2583(9)$ & $0.3405(6)$ & $0.087(8)$ & $0.043(7)$ & $0.068(8)$ & $0.006(5)$ & $-0.010(7)$ & $-0.013(6)$ \\
\hline $\mathrm{C}(9)$ & $4 a$ & $0.0446(3)$ & $-0.081(1)$ & $0.5068(5)$ & $0.048(5)$ & $0.055(6)$ & $0.056(5)$ & $0.006(5)$ & $-0.009(4)$ & $-0.016(7)$ \\
\hline$C(10)$ & $4 a$ & $0.0434(4)$ & $-0.2518(9)$ & $0.5106(7)$ & $0.074(8)$ & $0.069(8)$ & $0.087(9)$ & $0.008(5)$ & $0.022(8)$ & $0.002(8)$ \\
\hline$C(11)$ & $4 a$ & $0.1101(3)$ & $0.429(1)$ & $0.6224(4)$ & $0.052(5)$ & $0.049(5)$ & $0.051(5)$ & $0.002(4)$ & $-0.005(4)$ & $0.001(4)$ \\
\hline$C(12)$ & $4 a$ & $0.1417(3)$ & $0.497(1)$ & $0.6760(7)$ & $0.112(7)$ & $0.080(6)$ & $0.076(6)$ & $-0.017(5)$ & $-0.031(8)$ & $-0.016(7)$ \\
\hline$C(13)$ & $4 a$ & $-0.0768(3)$ & $0.4269(8)$ & $0.5074(4)$ & $0.051(5)$ & $0.046(5)$ & $0.047(4)$ & $0.007(4)$ & $0.009(4)$ & $0.001(4)$ \\
\hline$C(14)$ & $4 a$ & $-0.1313(3)$ & $0.497(1)$ & $0.5104(7)$ & $0.052(5)$ & $0.087(7)$ & $0.105(7)$ & $0.005(5)$ & $-0.004(6)$ & $0.023(7)$ \\
\hline$C(15)$ & $4 a$ & $0.1056(3)$ & $0.4369(9)$ & $0.3867(4)$ & $0.049(5)$ & $0.045(5)$ & $0.049(5)$ & $0.000(4)$ & $0.004(4)$ & $-0.001(4)$ \\
\hline$C(16)$ & $4 a$ & $0.1376(4)$ & $0.516(1)$ & $0.3398(6)$ & $0.116(9)$ & $0.074(8)$ & $0.068(7)$ & $0.009(7)$ & $0.010(7)$ & $-0.003(6)$ \\
\hline$C(17)$ & $4 a$ & $0.4141(3)$ & $0.2189(8)$ & $0.6759(5)$ & $0.057(5)$ & $0.051(5)$ & $0.050(4)$ & $-0.004(4)$ & $0.001(5)$ & $-0.009(5)$ \\
\hline$C(18)$ & $4 a$ & $0.4747(3)$ & $0.2005(9)$ & $0.6804(6)$ & $0.059(5)$ & $0.075(7)$ & $0.086(7)$ & $0.015(4)$ & $0.000(6)$ & $-0.005(7)$ \\
\hline$C(19)$ & $4 a$ & $0.2305(3)$ & $0.211(1)$ & $0.5464(4)$ & $0.044(5)$ & $0.059(5)$ & $0.051(6)$ & $0.007(5)$ & $0.003(4)$ & $-0.012(5)$ \\
\hline$C(20)$ & $4 a$ & $0.2013(3)$ & $0.183(1)$ & $0.4865(4)$ & $0.057(6)$ & $0.093(8)$ & $0.065(6)$ & $0.002(6)$ & $-0.013(4)$ & $-0.005(6)$ \\
\hline$C(21)$ & $4 a$ & $0.2906(3)$ & $0.6755(8)$ & $0.6704(5)$ & $0.046(4)$ & $0.048(5)$ & $0.043(4)$ & $-0.006(4)$ & $0.004(4)$ & $0.016(7)$ \\
\hline$C(22)$ & $4 a$ & $0.2894(3)$ & $0.8451(7)$ & $0.6720(6)$ & $0.084(5)$ & $0.042(4)$ & $0.065(5)$ & $0.002(5)$ & $0.008(6)$ & $0.005(8)$ \\
\hline$C(23)$ & $4 a$ & $0.2296(4)$ & $0.226(1)$ & $0.7959(4)$ & $0.048(5)$ & $0.071(6)$ & $0.040(5)$ & $-0.007(4)$ & $-0.001(4)$ & $-0.006(5)$ \\
\hline$C(24)$ & $4 a$ & $0.2009(4)$ & $0.210(1)$ & $0.8576(5)$ & $0.073(7)$ & $0.13(1)$ & $0.052(6)$ & $-0.017(7)$ & $0.007(5)$ & $0.000(7)$ \\
\hline
\end{tabular}

Acknowledgments. The authors thank the Education Office of Anhui Province, P. R. China, and the Natural Science Foundation of Hubei Province, P. R. China, for the research grants no. 2004kj300zd and no. 2003ABB010, respectively.

\section{References}

1. Csoregh, I.; Kierkegaard, P.; Norrestam, R.: Copper(I) tetraacetonitrile perchlorate. Acta Crystallogr. B31 (1975) 314-317.

2. Jones, P. G.; Bembenek, E.: Redetermination of the structure of tetrakis(acetonitrilo-N)silver(I) perchlorate at 173 K. Z. Kristallogr. 208 (1993) 213-218.

3. Jones, P. G.; Crespo, O.: Tetrakis(acetonitrile- $N$ )copper(I) tetrafluoroborate. Acta Crystallogr. C54 (1998) 18-20.
4. Yokota, Y.; Young Jr., V. G.; Verkade, J. G.: The homoleptic lithium complex. Acta Crystallogr. C55 (1999) 196-198.

5. Sheldrick, G. M.: SADABS. Program for Empirical Absorption Correction of Area Detector Data. University of Göttingen, Germany 1996.

6. Sheldrick, G. M.: SHELXTL V. 5.1. Software Reference Manual, Bruker AXS, Inc., Madison, Wisconsin, USA 1997. 